The Astrophysical Journal, 485:228-239, 1997 August 10

(C) 1997. The American Astronomical Society. All rights reserved. Printed in U.S.A.

\title{
THE PARKER INSTABILITY UNDER A LINEAR GRAVITY
}

\author{
Jongsoo Kim, ${ }^{1,2}$ Seung Soo Hong, ${ }^{2}$ AND Dongsu RYu ${ }^{3}$ \\ Received 1996 November 6; accepted 1997 March 13
}

\begin{abstract}
A linear stability analysis has been done to a magnetized disk under a linear gravity. We have reduced the linearized perturbation equations to a second-order differential equation that resembles the Schrödinger equation with the potential of a harmonic oscillator. Depending on the signs of energy and potential terms, eigensolutions can be classified into "continuum" and "discrete" families. When the magnetic field is ignored, the continuum family is identified as the convective mode, while the discrete family is identified as acoustic-gravity waves. If the effective adiabatic index $\gamma$ is less than unity, the former develops into the convective instability. When a magnetic field is included, the continuum and discrete families further branch into several solutions with different characters. The continuum family is divided into two modes: one is the original Parker mode, which is a slow MHD mode modulated by the gravity, and the other is a stable Alfvén mode. The Parker modes can be either stable or unstable depending on $\gamma$. When $\gamma$ is smaller than a critical value $\gamma_{\mathrm{cr}}$, the Parker mode becomes unstable. The discrete family is divided into three modes: a stable fast MHD mode modulated by the gravity, a stable slow MHD mode modulated by the gravity, and an unstable mode that is also attributed to a slow MHD mode. The unstable discrete mode does not always exist. Even though the unstable discrete mode exists, the Parker mode dominates it if the Parker mode is unstable. However, if $\gamma \geq \gamma_{\mathrm{cr}}$, then the discrete mode could be the only unstable one. When $\gamma$ is equal $\gamma_{\text {cr }}$, the minimum growth time of the unstable discrete mode is $1.3 \times 10^{8} \mathrm{yr}$, with a corresponding length scale of $2.4 \mathrm{kpc}$. It is suggestive that the corrugated features seen in the Galaxy and external galaxies are related to the unstable discrete mode.
\end{abstract}

Subject heading: accretion, accretion disks — instabilities - ISM: magnetic fields - MHD

\section{INTRODUCTION}

Parker (1966) demonstrated that the equilibrium state of interstellar gas, the magnetic field, and cosmic rays under the vertical gravity could be unstable to long-wavelength perturbations along the direction of the equilibrium magnetic field. Since then, many authors have studied the details of this instability. For instance, two-dimensional, nonlinear equilibrium states resulting from the Parker instability under a uniform gravity were derived by Mouschovias (1974). The effects of rotation (Shu 1974; Zweibel \& Kulsrud 1975; Foglizzo \& Tagger 1994), nonuniform gravity (Horiuchi et al. 1988; Matsumoto et al. 1988; Giz \& Shu 1993), and skewed magnetic field (Hanawa, Matsumoto, \& Shibata 1992) on the Parker instability were discussed. The Parker instability was considered to be a plausible mechanism for the formation of large-scale interstellar clouds (Appenzeller 1974; Mouschovias, Shu, \& Woodward 1974; Blitz \& Shu 1980; Shibata \& Matsumoto 1991; Gomez de Castro \& Pudritz 1992), a possible process for a dynamo in accretion disks (Tout \& Pringle 1992), and the force driving emergent flux tubes, which are closely related to the active phenomena of the Sun (Shibata et al. 1989a, 1989b; Kaisig et al. 1990; Nozawa et al. 1992).

The original analysis of the instability by Parker (1966) was performed under a uniform gravity, although he commented on the effects of a nonuniform, linear gravity. Since the uniform gravity has a jump at the midplane, the physi-

\footnotetext{
${ }^{1}$ Korea Astronomy Observatory, San 36-1, Whaam-Dong, YusongKu, Taejon 305-348, Korea; jskim@ hanul.issa.re.kr.

${ }^{2}$ Department of Astronomy, Seoul National University, Seoul 151-742, Korea; sshong@astroism.snu.ac.kr.

${ }^{3}$ Department of Astronomy and Space Science, Chungnam National University, Taejon 305-764, Korea; and Department of Astronomy, University of Washington, Seattle; ryu@sirius.chungnam.ac.kr.
}

cal quantities should have a cusp. Because of this, the perturbations in the upper hemisphere cannot communicate with those in the lower hemisphere. Thus, Parker's analysis should have been restricted to the "symmetric" mode, where the vertical velocity perturbation vanishes at the midplane. In order to remove this difficulty, a nonuniform gravity that changes smoothly at the midplane should be introduced.

Giz \& Shu (1993) considered the Parker instability under a gravity whose functional form is $-g_{0} \tanh \left(z / 2 H_{*}\right)$, where $g_{0}$ and $H_{*}$ are constants, and $z$ is the vertical distance from the midplane. With the scale height of stars for $H_{*}$, this gravity closely represents the Galactic gravitational field. In the limit $|z| \rightarrow \infty$ it is reduced to a uniform gravity, while in $|z| \rightarrow 0$ it is reduced to a linear gravity. With this gravity, Giz \& Shu (1993) obtained a dispersion relation that matches that under the uniform gravity. They called it the "continuum" family. But, in addition, they found a dispersion relation that has no correspondence in the uniform gravity. They called the latter the "discrete" family. Before Giz \& Shu (1993), Horiuchi et al. (1988) considered the Parker instability under a point mass-dominated gravity, which is continuous across the midplane. It is a good model for the gravitational field in accretion disks. They considered isothermal perturbations with the effective adiabatic index $\gamma=1$ and obtained dispersion relations for both the symmetric and antisymmetric modes. Their solutions correspond to the continuum family by the Giz \& Shu (1993) classification. They did not see the solutions of the discrete family, since their analysis was restricted to isothermal perturbations.

In this paper, we consider a linear gravity whose functional form is $-g^{\prime} z$, where $g^{\prime}$ is a constant. The linear gravity represents fairly well the $z$-dependence of the Galactic gravity up to $500 \mathrm{pc}$ (Oort 1965), as well as the "vertical" 
gravity of accretion disks. It is also easier to handle than those introduced by Giz \& Shu (1993) and Horiuchi et al. (1988). Although Parker (1966) considered a linear gravity, all he did was show that the equilibrium state of an interstellar gas-field system also becomes unstable under it. Here we investigate the resulting eigensolutions in detail: we classify them into the continuum and discrete families, discuss their nature, and give criteria for the existence of the unstable continuum and discrete families. Although the existence of the discrete family was already discussed by Giz \& Shu (1993), our work will serve to get a better insight into its nature and its possible roles in astrophysics.

Several authors have reported that there exist corrugations in our Galaxy (Quiroga \& Schlosser 1977; Lockman 1977; Spicker \& Feitzinger 1986; Alfaro, Cabrera-Caño, \& Delgado 1992; Malhotra 1994) and external galaxies (Florido et al. 1991) as well. Nelson (1976) suggested that the corrugations may be a manifestation of hydrodynamic acoustic-gravity waves. The difficulties with Nelson's suggestion are that the characteristic scale does not come out naturally and that the structures formed by the acousticgravity waves should be oscillatory and transient. Instead, in this paper we suggest that the corrugations may be formed as the result of the unstable discrete mode.

The plan of the paper is as follows. In $\S 2$ the classification of the eigensolutions into the continuum and discrete families is given. In $\S 3$ the dispersion relations of the eigensolutions are analyzed in detail. We first consider the dispersion relations of nonmagnetized disks and then move to those of magnetized disks. In $\S 4$ a summary and discussion are given. The derivation of eigenvalue equations and the method we used to obtain the dispersion relation of the continuum family are described in Appendices A and B.

\section{CONTINUUM AND DISCRETE FAMILIES}

We consider the stability of a disk composed of gas, magnetic field, and cosmic rays under a linear gravity. For the unperturbed equilibrium state, we follow Parker (1966) in assuming that the gas is isothermal and that the ratio of magnetic pressure to gas pressure, $\alpha$, and the ratio of cosmic-ray pressure to gas pressure, $\beta$, are spatially constant. Then, the unperturbed gas density, gas pressure, cosmic-ray pressure, and magnetic pressure are given by a Gaussian distribution in $z$. The equations for the stability analysis are obtained by introducing an infinitesimal perturbation to the unperturbed equilibrium state. Those equations can be combined into a single eigenvalue equation, which is a second-order differential equation. The detailed manipulation of the equations is described in Appendix A. The resulting eigenvalue equation is

$$
\frac{d^{2} \psi}{d \zeta^{2}}+\left(E-V_{0} \zeta^{2}\right) \psi=0
$$

where $E$ and $V_{0}$ are defined by

$$
\begin{aligned}
& E \equiv \frac{1}{2}+ \\
& \frac{\Omega^{4}-\left[(1+\alpha+\beta)+(2 \alpha+\gamma)\left(v_{x}^{2}+v_{y}^{2}\right)\right] \Omega^{2}+2 \alpha \gamma\left(v_{x}^{2}+v_{y}^{2}\right) v_{y}^{2}}{(2 \alpha+\gamma) \Omega^{2}-2 \alpha \gamma v_{y}^{2}},
\end{aligned}
$$

$$
\begin{aligned}
V_{0} \equiv & \frac{1}{4}-\frac{2 \alpha(1+\alpha+\beta) v_{x}^{2} \Omega^{2}}{\left(\Omega^{2}-2 \alpha v_{y}^{2}\right)\left[(2 \alpha+\gamma) \Omega^{2}-2 \alpha \gamma v_{y}^{2}\right]} \\
& +\frac{(1+\alpha+\beta)(1+\alpha+\beta-\gamma)\left(v_{x}^{2}+v_{y}^{2}\right)}{(2 \alpha+\gamma) \Omega^{2}-2 \alpha \gamma v_{y}^{2}} .
\end{aligned}
$$

Here $\zeta$ is a normalized $z$-coordinate, $v_{x}$ and $v_{y}$ are normalized radial and azimuthal wavenumbers, $\Omega$ is a normalized angular frequency, and $\psi$ is a quantity proportional to the normalized vertical velocity multiplied by the square root of the unperturbed normalized gas density (see eqs. [A28] and [A30]). It should be pointed out that $\psi^{2}$ is the kinetic energy density associated with the motion in the $z$-direction. Normalization units are the disk scale height $H$, sound speed of unperturbed state $a_{s}$, and unperturbed gas density at the midplane $\rho_{0}(0)$. Because of the simple functional form of the gravity we used, we could get an eigenvalue equation that resembles the Schrödinger equation with the potential of a harmonic oscillator. As the boundary condition, we assume that $\psi$ stays finite as $\zeta \rightarrow \infty$. That is, the energy density perturbation does not diverge at infinite.

The solutions of the eigenvalue equation can be classified into four different types by the signs of $E$ and $V_{0}$ (Fig. 1). If $E>0$ and $V_{0}<0$ so that $E$ is greater than $V(\zeta)=V_{0} \zeta^{2}$ in the whole range of $\zeta,-\infty<\zeta<+\infty$, then all the positive values are allowed as the eigenvalue $E$ (type I). In this case, the eigenfunction $\psi$ oscillates as $|\zeta|$ increases. If $E<0$ and $V_{0}<0$ so that $E$ is smaller than $V(\zeta)$ in a finite interval that includes $\zeta=0$ and greater than $V(\zeta)$ in the rest, then all the negative values are allowed as the eigenvalue (type II). The eigenfunction declines exponentially in the finite interval but stays oscillatory outside it. If $E>0$ and $V_{0}>0$ so that $E$ is greater than $V(\zeta)$ in a finite interval but smaller than $V(\zeta)$ in the rest, then only discrete positive values are allowed as the eigenvalue (type III). The eigenfunction oscil-
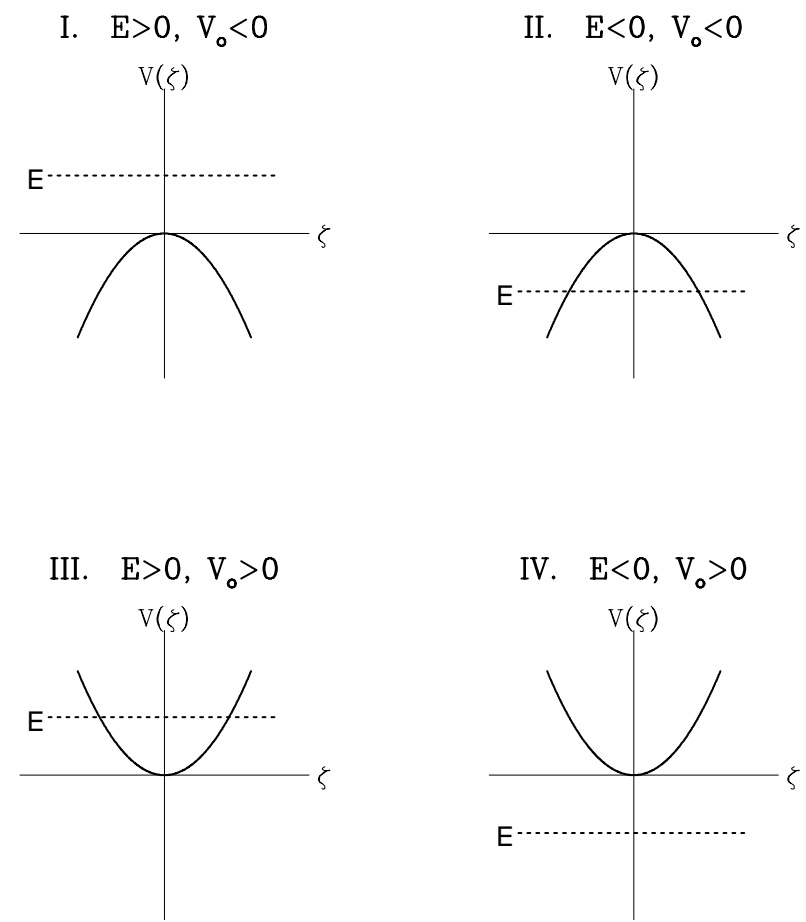

FIG. 1.-By the signs of $E$ and $V_{0}$, the solutions of the eigenvalue equation (1) are classified into four types. For types I and II, the solutions belong to the continuum family. For type III the solutions belong to the discrete family. For type IV, the solutions do not satisfy the imposed boundary condition. 
lates in the interval but declines exponentially outside it. If $E<0$ and $V_{0}>0$ so that $E$ is smaller than $V(\zeta)$ in the whole interval, then there are no physically meaningful solutions satisfying the boundary condition (type IV). With the tangent hyperbolic gravity, Giz \& Shu (1993) also obtained an equation similar to equation (1) and classified its solutions into the continuum and discrete families. According to the Giz \& Shu (1993) classification, the solutions of type I and II correspond to the continuum family, and those of type III to the discrete family.

\subsection{Continuum Family}

For the continuum family with negative $V_{0}$, we introduce a new variable $\xi$ defined by $\xi=2^{1 / 2}\left(-V_{0}\right)^{1 / 4} \zeta$ and transform equation (1) into that for the parabolic cylinder function (Abramowitz \& Stegun 1970, p. 685):

$$
\begin{gathered}
\frac{d^{2} \psi}{d \xi^{2}}+\left(\frac{1}{4} \xi^{2}-a\right) \psi=0, \\
a \equiv \frac{1}{2} \frac{-E}{\sqrt{-V_{0}}} .
\end{gathered}
$$

As $|\xi|$ increases, the parabolic cylinder function oscillates in the interval between nodal points. For $|\xi| \rightarrow \infty, \psi \propto \exp$ $\left( \pm i \xi^{2}\right) / \xi^{1 / 2}$. Thus, the energy density perturbation of the continuum family decreases as $1 /|\xi|$ at large $|\xi|$. It is interesting to notice that, for $|\xi| \rightarrow \infty$, the $z$ wavelength decreases as $1 /|\xi|$; hence, the group velocity along the $z$ direction increases as $|\xi|$. Consequently, the energy flux of the continuum family stays constant at large $|\xi|$.

The dispersion relations for the symmetric mode can be calculated by considering the lower boundary condition at the midplane, $\psi(\xi=0)=0$, while the dispersion relations for the antisymmetric mode by taking $d \psi(\xi=0) / d \xi=0$ (see Appendix B). With the condition $\psi \xi^{1 / 2}=$ finite at $|\xi| \rightarrow \infty$, there exists, for a given pair of wavenumbers $v_{x}^{2}$ and $v_{y}^{2}$, an interval of $\Omega^{2}$ values. Here, we find solutions with the energy density perturbation zero at the upper boundary, which is identified to the first nodal point from the midplane, $\xi_{\text {node }}$. Then, the value of $\Omega^{2}$ is uniquely determined for the given nodal point. Since the nodal point indicates the position where the vertical velocity goes to zero, the distance to this nodal point may be considered as an "effective" wavelength along the $z$-direction. A method to find the value of $\Omega^{2}$ with a chosen nodal point is described in Appendix B.

\subsection{Discrete Family}

For the discrete family with positive $V_{0}$ and positive $E$, equation (1) has exactly the same form as the Schrödinger equation with the potential of a harmonic oscillator. The solution of the harmonic oscillator problem is well known: the eigenfunctions are

$$
\psi_{n}(\zeta)=\frac{\sqrt[8]{V_{0}}}{\sqrt[4]{\pi} \sqrt{2^{n} n !}} H_{n}\left(\sqrt[4]{V_{0}} \zeta\right) \exp \left(-\frac{\sqrt{V_{0}}}{2} \zeta^{2}\right),
$$

and the corresponding eigenvalues are

$$
\frac{E}{\sqrt{V_{0}}}=2 n+1,
$$

where $H_{n}$ is the Hermite polynomial of degree $n$, and $n$ takes values $0,1,2, \ldots$ In this case, $\psi$ decreases exponentially at large $|\zeta|$, so the energy density perturbation of the discrete family does as well.

The eigenfunctions for $n=1,3, \ldots$ that have $\psi=0$ at $\zeta=0$ represent the symmetric mode, while the eigenfunctions for $n=0,2, \ldots$ that have $d \psi / d \zeta=0$ at $\zeta=0$ represent the antisymmetric mode. The eigenvalue relation of equation (7) gives the dispersion relation, i.e., $\Omega^{2}$ as a function of $v_{x}^{2}, v_{y}^{2}$, and $n$. Here, $n$ indicates the number of nodal points along the $z$-direction, so it may be considered as the "effective" wavenumber in the $z$-direction.

\section{WAVES AND INSTABILITIES}

In this section, we explore the nature of the continuum and discrete families in detail by analyzing both the stable (waves) modes and the unstable (instability) modes. We first consider the case of nonmagnetized disks, then include a magnetic field and cosmic rays.

\subsection{Nonmagnetized Disks}

Without a magnetic field, the cosmic rays are ignored, so $\alpha=\beta=0$. Without a magnetic field, there is no preferred direction in the $x-y$ plane, and we can set $v_{x}=0$ without losing any generality. Then, $E$ and $V_{0}$ become

$$
\begin{gathered}
E=\frac{1}{2}+\frac{\Omega^{2}-\left(1+\gamma v_{y}^{2}\right)}{\gamma}, \\
V_{0}=\frac{1}{4}+\frac{(1-\gamma) v_{y}^{2}}{\gamma \Omega^{2}} .
\end{gathered}
$$

Equation (1) with the above $E$ and $V_{0}$ is exactly the one Nelson (1976) derived to study hydrodynamic acousticgravity waves under a linear gravity.

In the case of $\gamma=1$ for isothermal perturbations, $V_{0}=$ $\frac{1}{4}$ is always positive. So, equation (1) has only two types of solutions, type III and type IV, depending on the sign of $E$. The locus of $E=0$ is drawn with a dashed line in the phase space of $v_{y}^{2}$ and $\Omega^{2}$ in Figure $2 a$. We are interested only in the upper region of the line with $E>0$, where the solutions of type III exist. The eigenfunctions of the solutions in this region are

$$
\psi(\zeta)=\frac{1}{\sqrt[4]{2 \pi}} \frac{1}{\sqrt{2^{n} n !}} H_{n}\left(\frac{\zeta}{\sqrt{2}}\right) \exp \left(-\frac{\zeta^{2}}{4}\right),
$$

and the corresponding dispersion relations are

$$
\Omega^{2}=v_{y}^{2}+n+1 .
$$

Equation (11) reduces to the dispersion relation $\Omega^{2} \simeq v_{y}^{2}$ of sound waves for $v_{y}^{2} \gg n+1$. Thus, the dispersion relations are of the acoustic-gravity waves, which have the characteristics of sound waves modulated by the vertical gravity, as was pointed out by Nelson (1976). In Figure $2 b$, the dispersion relations of the acoustic-gravity waves with $n=0$, $1, \ldots, 6$ are plotted with solid lines. In this case, no convective mode exits, so all the modes are stable.

If $\gamma \neq 1$, then the signs of both $E$ and $V_{0}$ can be positive or negative. In Figures $3 a$ and $4 a$, three loci of $E=0, V_{0}=0$, and $\Omega^{2}=0$ are drawn for $\gamma=0.8$ and 1.3 with a dashed line $(E=0)$, a dotted line $\left(V_{0}=0\right)$, and a long-dashed line $\left(\Omega^{2}=0\right)$. The phase space of $v_{y}^{2}$ and $\Omega^{2}$ is divided into four regions by the three loci, which are identified as those of the types II, III, and IV solutions. We are interested only in the regions of the type II (continuum family) and the type III (discrete family) solutions. In Figures $3 b$ and $4 b$, we plot the 

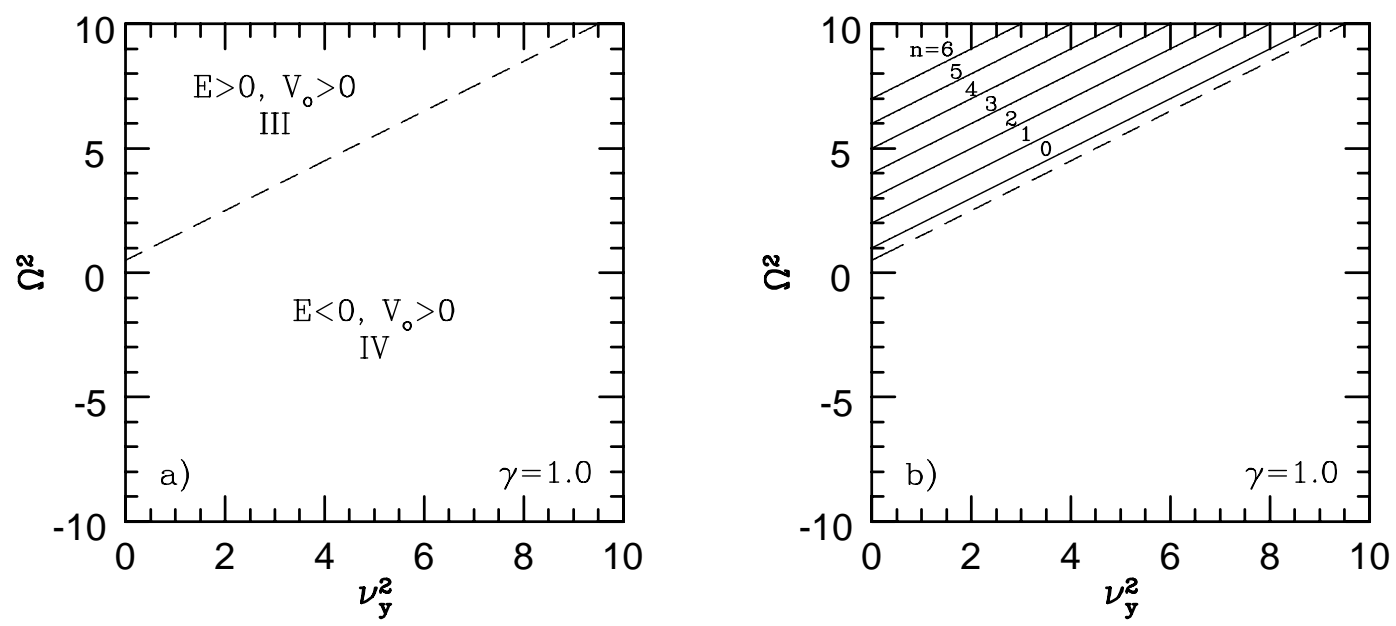

Fig. 2.-(a) Division of the phase space of $v_{y}^{2}$ and $\Omega^{2}$ into regions with the solutions of different types for a nonmagnetized disk with $\gamma=1$. The dashed line is the locus of $E=0$. (b) Dispersion relations of the discrete family solutions with $n=0,1,2, \ldots, 6$ in the region of type III.
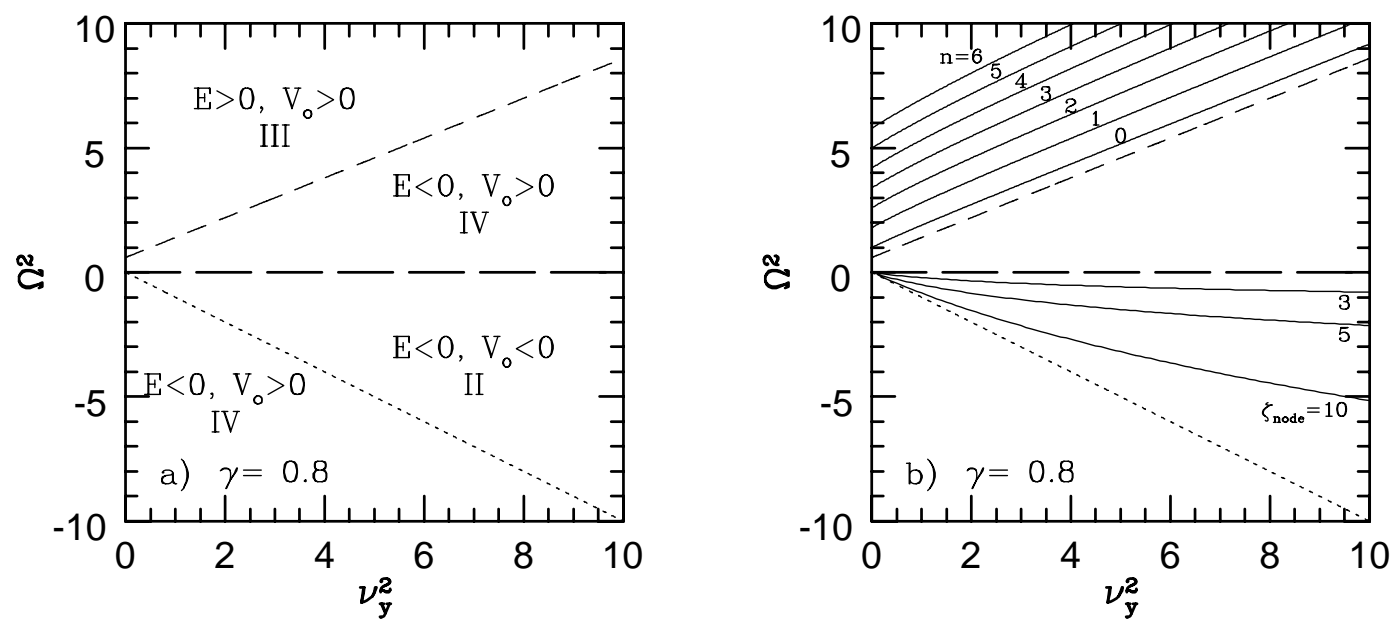

FIG. 3.-(a) Division of the phase space of $v_{y}^{2}$ and $\Omega^{2}$ into regions with the solutions of different types for a nonmagnetized disk with $\gamma=0.8$. Three loci of $E=0$ (dashed line), $V_{0}=0$ (dotted line), and $\Omega^{2}=0$ (long-dashed line) are drawn. (b) Dispersion relations of the discrete family solutions with $n=0,1,2, \ldots, 6$ in the region of type III, and the dispersion relations of the continuum family solutions with nodal points $\zeta_{\text {node }}=3,5,10$ in the region of type II.
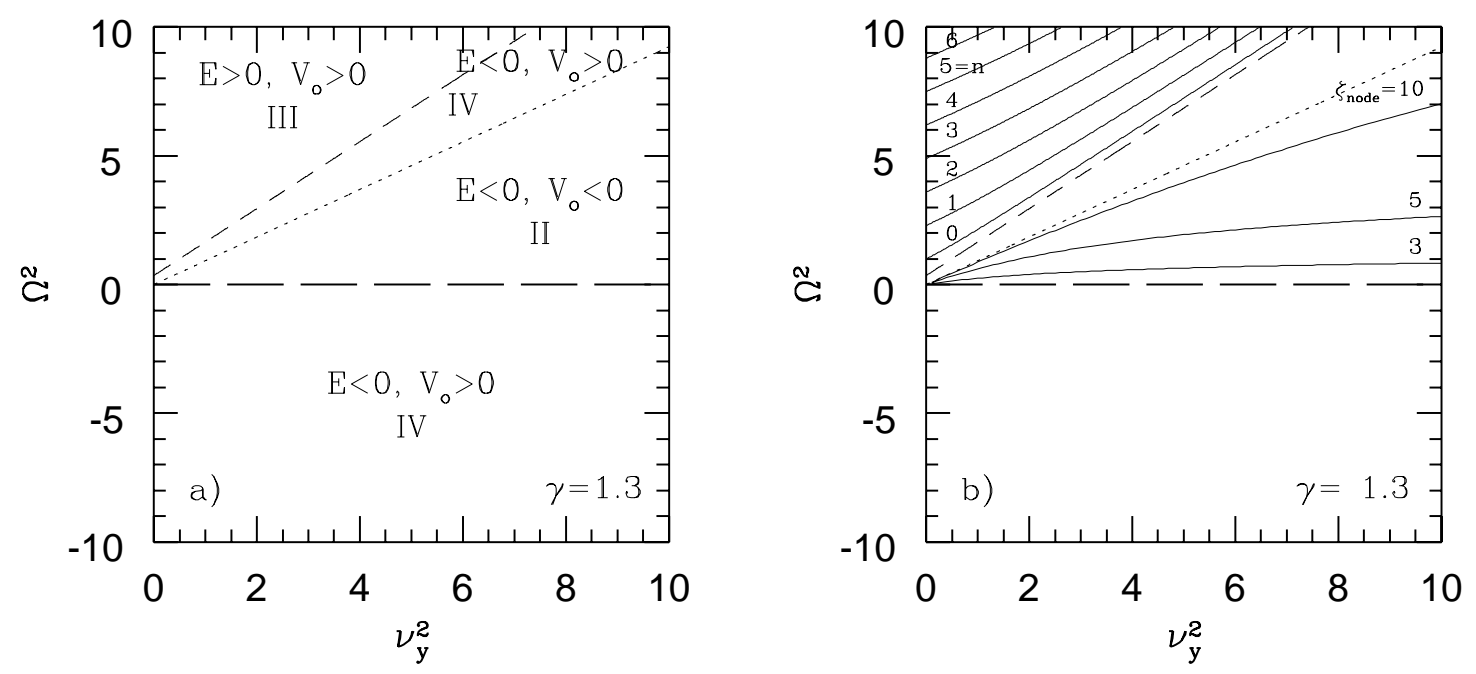

FIG. 4.-(a) Division of the phase space of $v_{y}^{2}$ and $\Omega^{2}$ into regions with the solutions of different types for a nonmagnetized disk with $\gamma=1.3$. Three loci of $E=0$ (dashed line), $V_{0}=0$ (dotted line), and $\Omega^{2}=0$ (long-dashed line) are drawn. (b) Dispersion relations of the discrete family solutions with $n=0,1,2, \ldots, 6$ in the region of type III, and the dispersion relations of the continuum family solutions with nodal points $\zeta_{\text {node }}=3,5,10$ in the region of type II. 
dispersion relations of the discrete family solutions with $n=0,1, \ldots, 6$, which are obtained from equation (7), and the dispersion relations of the continuum family solutions with nodal points at $\zeta_{\text {node }}=3,5,10$, which are computed by the method described in Appendix B.

The solutions of the discrete family, which are identified as acoustic-gravity waves, always have positive $\Omega^{2}$ values, so they are stable. On the other hand, the solutions of the continuum family, which can be identified as the convective mode, are stable if $\gamma>1$, but unstable if $\gamma<1$, developing into the convective instability (Ryu \& Goodman 1992). The mode with a larger $\zeta_{\text {node }}$ is more unstable, because the gravity is a linearly increasing function of $\zeta$.

In the case of a uniform gravity, the square of the maximum growth rate of the convective instability is $\Omega^{2}=-(1-\gamma) / \gamma(\operatorname{Ryu} \&$ Goodman 1992). For $\gamma=0.8$, it is -0.25 . Except at small wavenumbers, the growth rate of the convective instability under the linear gravity in Figure $3 b$ is larger than the maximum growth rate under the uniform gravity.

The dispersion relations of the continuum family solutions shown in Figures $3 b$ and $4 b$ are for the symmetric mode. In order to see the effects of the boundary condition at the midplane, we have also calculated the dispersion relations of the antisymmetric mode. Figure 5 shows the resulting dispersion relations with nodal points at $\zeta_{\text {node }}=2$ and 3. Solid lines represent the dispersion relations of the symmetric mode, and dotted lines those of the antisymmetric mode. The growth rates of both modes are nearly the same, unless $\zeta_{\text {node }} \ll 1$. This is true even if the magnetic field is included. The reason for this is that the gravity is small near the midplane. Thus, the details around the midplane are not important unless $\zeta_{\text {node }} \ll 1$. In the rest of the paper, we then present only the dispersion relation of the symmetric mode for the continuum family solutions.

\subsection{Magnetized Disks}

\subsubsection{Limit of $H \rightarrow \infty$}

Taking the limit $H \rightarrow \infty$ means also making $g^{\prime} \rightarrow 0$. So the problem reduces to that of finding the dispersion rela-

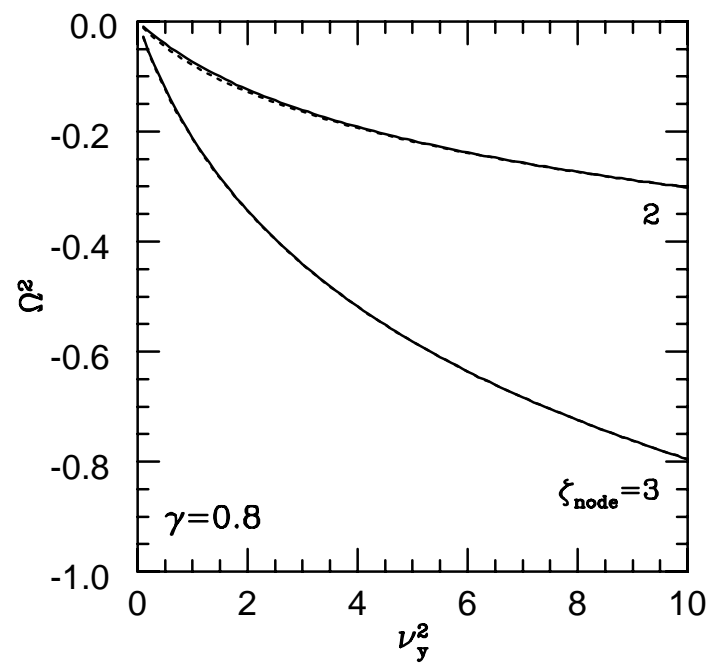

FIG. 5.-Dispersion relations of the symmetric mode solutions (solid lines) and the antisymmetric mode solutions (dotted lines) of the continuum family with nodal points $\zeta_{\text {node }}=2$ and 3 for a nonmagnetized disk with $\gamma=0.8$. tion in a uniform medium without gravity. Since the perturbation of cosmic-ray pressure is zero in the uniform medium (see [A26]), we should get the dispersion relations for three magnetohydrodynamic (MHD) waves. From (A18) to (A25), by setting $d / d z=0$, we have

$$
\begin{aligned}
& \left(\frac{\omega^{2}}{a_{s}^{2}}-2 \alpha k_{y}^{2}\right) \\
& \quad \times\left[\frac{\omega^{4}}{a_{s}^{4}}-(2 \alpha+\gamma)\left(k_{x}^{2}+k_{y}^{2}\right) \frac{\omega^{2}}{a_{s}^{2}}+2 \alpha \gamma\left(k_{x}^{2}+k_{y}^{2}\right) k_{y}^{2}\right] \Psi=0,
\end{aligned}
$$

where $a_{s}$ is the isothermal sound speed. The above dispersion relation can be decoupled into one for the Alfvén waves

$$
\omega^{2}-v_{\mathrm{A}}^{2} k_{y}^{2}=0
$$

and the other for the slow and the fast MHD waves

$$
\omega^{4}-\left(v_{\mathrm{A}}^{2}+c_{s}^{2}\right)\left(k_{x}^{2}+k_{y}^{2}\right) \omega^{2}+v_{\mathrm{A}}^{2} c_{s}^{2} k_{y}^{2}\left(k_{x}^{2}+k_{y}^{2}\right)=0 .
$$

Here, $v_{\mathrm{A}}=2 \alpha a_{s}^{2}$ and $c_{s}^{2}=\gamma a_{s}^{2}$ are the Alfvén and adiabatic sound speeds.

$$
\text { 3.2.2. Case of } v_{x}=0
$$

It is known that the growth rate of the Parker instability is larger for perturbations with larger $x$ wavenumbers (Parker 1967). However, in this subsection we first consider the case of $v_{x}=0$ in detail, since the equations involved are much simpler, and yet most important physical aspects are included. We comment briefly on the general case of $v_{x} \neq 0$ in the next subsection.

In the case of $v_{x}=0$, the $x$-components of velocity and magnetic field perturbations are decoupled from the rest and result in Alfvén waves with the dispersion relation (13) (see eqs. [A19] and [A22]). So, with the rest of the perturbation equations, we get the eigenvalue equation (1) with $E$ and $V_{0}$ given by

$$
\begin{gathered}
E=\frac{1}{2}+\frac{\Omega^{4}-\left[(1+\alpha+\beta)+(2 \alpha+\gamma) v_{y}^{2}\right] \Omega^{2}+2 \alpha \gamma v_{y}^{4}}{(2 \alpha+\gamma) \Omega^{2}-2 \alpha \gamma v_{y}^{2}}, \\
V_{0}=\frac{1}{4}+\frac{(1+\alpha+\beta)(1+\alpha+\beta-\gamma) v_{y}^{2}}{(2 \alpha+\gamma) \Omega^{2}-2 \alpha \gamma v_{y}^{2}}
\end{gathered}
$$

In order to classify its solutions, we draw in Figure $6 a$ the loci of $E=0, V_{0}=0$, and $(2 \alpha+\gamma) \Omega^{2}-2 \alpha \gamma v_{y}^{2}=0$ with two dashed lines, a dotted line, and a long-dashed line, respectively, for $\alpha=1, \beta=0$, and $\gamma=1$. Since $E=0$ reduces to a quadratic equation of $\Omega^{2}$, there exist two branches, which are labeled by $E^{+}=0$ (the positive branch) and $E^{-}=0$ (the negative branch). The loci of $E^{-}=0$ and $(2 \alpha+\gamma) \Omega^{2}$ $-2 \alpha \gamma v_{y}^{2}=0$ intersect at the point where $v_{y}^{2}=(2 \alpha+\gamma)$ $(1+\alpha+\beta) /(2 \alpha \gamma)$ and $\Omega^{2}=1+\alpha+\beta$. The intersection point exists also in the case of $v_{x}^{2} \neq 0$, and its position is determined only by $\alpha, \beta$, and $\gamma$. Thus, its location for the case of $v_{x}^{2} \neq 0$ is the same as that for the case of $v_{x}^{2}=0$. The four loci divide the phase space of $v_{y}^{2}$ and $\Omega^{2}$ into six regions, which are identified in Figure $6 a$ by the type of solutions. The continuum family solutions exist in the regions of type I and II, and the discrete family solutions in type III. Figure $6 b$ shows the dispersion relations of the discrete family solutions with $n=0,1,2$ and those of the continuum family solutions with nodal points at $\zeta_{\text {node }}=3$, 5,10 . 

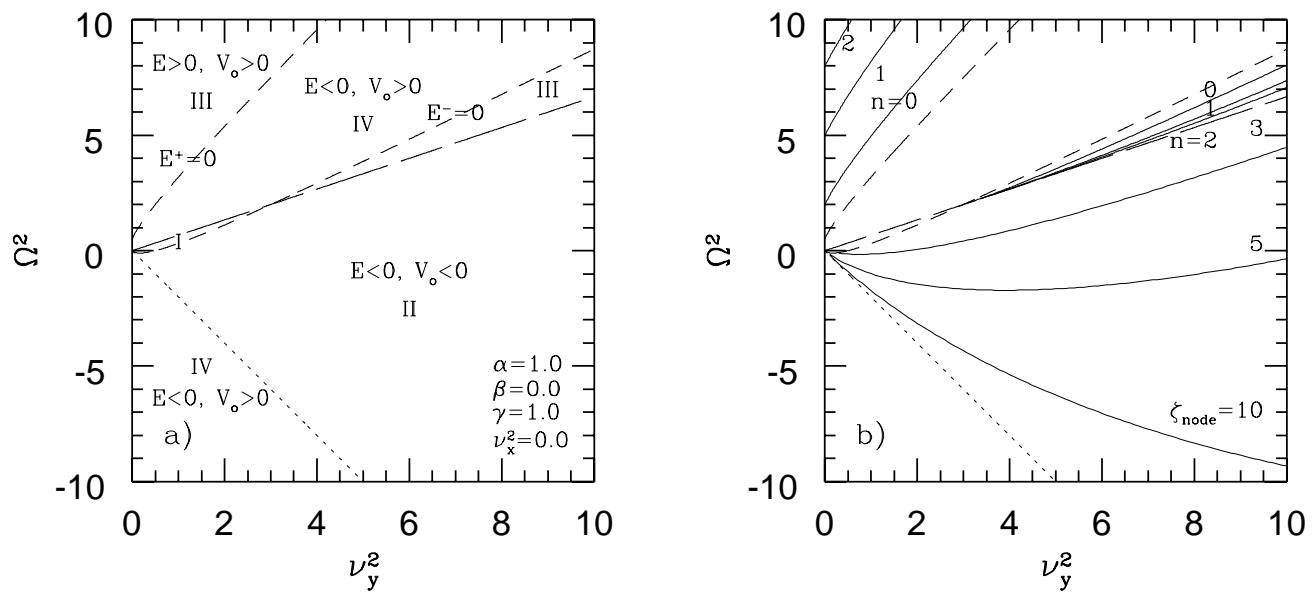

Fig. 6.-(a) Division of the phase space of $v_{y}^{2}$ and $\Omega^{2}$ into regions with the solutions of different types for a magnetized disk with $\alpha=1, \beta=0, \gamma=1$, and $v_{x}=0$. The loci of $E=0$ (dashed lines), $V_{0}=0$ (dotted line), and $(2 \alpha+\gamma) \Omega^{2}-2 \alpha \gamma v_{y}^{2}=0$ (long-dashed line) are drawn. (b) Dispersion relations of the discrete family solutions with $n=0,1,2$ in the regions of type III, and the dispersion relations of the continuum family solutions with nodal points $\zeta_{\text {node }}=3,5,10$ in the regions of types I and II.

The continuum family solutions correspond to those of the original Parker instability and are identified as a slow MHD mode modulated by the gravity (Parker 1966; Shu 1974). For the same nodal point and the same parameters $(\alpha, \beta, \gamma)$, the growth rate of the continuum family is larger under a linear gravity than under a uniform gravity. A full comparative study of the growth rate under different models of gravity will be given in a separate paper.

The discrete family solutions in the upper left region of Figure $6 b$ are MHD counterparts of Nelson's acousticgravity waves, which are identified as fast MHD waves modulated by gravity. The discrete family solutions in the region enclosed by the loci of $E^{-}=0$ and $(2 \alpha+\gamma) \Omega^{2}$ $-2 \alpha \gamma v_{y}^{2}=0$ represent a new mode, which was not discovered in any previous work. They are always stable, since $\Omega^{2}>0$ at the intersection point of the loci. On the following grounds, we identify them as slow MHD waves modulated by gravity. For $v_{y} \rightarrow \infty$ and $n \rightarrow \infty$, the dispersion relation of the discrete family becomes

$$
\Omega^{4}-(2 \alpha+\gamma)\left(v_{y}^{2}+v_{z}^{2}\right) \Omega^{2}+2 \alpha \gamma\left(v_{y}^{2}+v_{z}^{2}\right) v_{y}^{2}=0,
$$

if we set $2\left(V_{0}\right)^{1 / 2} n=v_{z}^{2}$ (see eqs. [7], [15], and [16]). Note that $V_{0}$ becomes constant in this limit. Equation (17) is the dispersion relation for the fast and slow waves (see eq. [14]). The fast waves correspond to the solutions in the upper left region, while the slow waves to those in the region enclosed by loci of $E^{-}=0$ and $(2 \alpha+\gamma) \Omega^{2}-2 \alpha \gamma v_{y}^{2}=0$.

If the slope of the locus of $V_{0}=0$ in the phase space of $v_{y}^{2}$ and $\Omega^{2}$ is equal to or greater than zero, the continuum family solutions are stabilized. For given $\alpha$ and $\beta$, the critical value of the adiabatic index, $\gamma_{\mathrm{cr}}$, which gives zero slope, is

$$
\gamma_{\mathrm{cr}}=\frac{(1+\alpha+\beta)^{2}}{1+(3 / 2) \alpha+\beta} .
$$

Note that this is same as the critical value for the case of a uniform gravity (Parker 1966). To see the nature of the solutions in the case of $\gamma \geq \gamma_{\mathrm{cr}}$, we draw in Figure $7 a$ the loci of $E=0, V_{0}=0$, and $(2 \alpha+\gamma) \Omega^{2}-2 \alpha \gamma v_{y}^{2}=0$ for $\alpha=1$, $\beta=0$, and $\gamma=\gamma_{\mathrm{cr}}$. Figure $7 b$ presents the dispersion relations of the discrete family solutions with $n=0,1,2$ and those of the continuum family solutions with nodal points at $\zeta_{\text {node }}=3,5,10$. As in the case of Figure 6, there are solutions of the continuum family in the regions of types I and II, but now they are stable with $\Omega^{2} \geq 0$. Also, there are solutions of two stable discrete modes in the upper left region and in the region enclosed by loci of $E^{-}=0$ and $(2 \alpha+\gamma) \Omega^{2}-2 \alpha \gamma v_{y}^{2}=0$. However, there is another region of type III enclosed by loci of $E^{-}=0$ and $V_{0}=0$, as can be seen in the enlarged plot of Figure 7c. The solutions of the discrete mode in this region are unstable with $\Omega^{2}<0$. There are an infinite number of unstable solutions with $n=0,1,2$, $\ldots$, and Figure $7 d$ shows the dispersion relations with $n=0$, $1,2$.

Figure 8 shows the eigenfunctions of the unstable discrete mode solutions with $n=0,1,2$ for $\alpha=1, \beta=0, \gamma=\gamma_{\mathrm{cr}}$, and $v_{x}=0$. For a given $n$, the most unstable growth rate and its azimuthal wavenumber are used. The eigenfunctions of odd $n$ values are of the symmetry mode with $\psi(0)=0$, while those of even $n$ values are of the antisymmetry mode with $d \psi(0) / d \zeta=0$. As was noted in $\S 2.2$, the energy density perturbation of the discrete mode solutions is concentrated around $\zeta=0$. Since the stable discrete mode solutions in the top left region are identified as the fast MHD mode modulated by gravity, we may attribute the unstable discrete solutions to the slow MHD mode modulated by gravity as the stable discrete solutions in the region enclosed by loci of $E^{-}=0$ and $(2 \alpha+\gamma) \Omega^{2}-2 \alpha \gamma v_{y}^{2}=0$.

The unstable discrete mode does not always exist. Its existence depends on whether the two loci of $E^{-}=0$ and $V_{0}=0$ meet at two points: one at the origin $\left(v_{y}^{2}=0, \Omega^{2}=0\right)$ and the other denoted by $\left(v_{y, \text { int }}^{2}, \Omega_{\text {int }}^{2}\right)$. For $\beta=0$, Figure $9 a$ shows the equi- $v_{y, \text { int }}^{2}$ contours on the $[\alpha, \gamma]$ plane, and Figure $9 b$ shows the equi- $\Omega_{\text {int }}^{2}$ contours. The unstable discrete mode exists if $\gamma$ has a value in the region between the two lines of $\Omega_{\mathrm{int}}^{2}=0$ in Figure $9 b$, or if $1<\gamma<\gamma_{\text {int }}$. Here, $\gamma_{\text {int }}$ is a function of $\alpha$ in this case of $\beta=0$ and $v_{x}=0$, but it depends on other parameters, too, in general. If a medium has $1<\gamma<\min \left(\gamma_{\mathrm{cr}}, \gamma_{\text {int }}\right)$, both the unstable continuum and discrete modes exist. The growth rate of the unstable continuum mode, especially at small wavelengths, is always larger than that of the unstable discrete mode, making the continuum mode dominate over the discrete mode. On the 

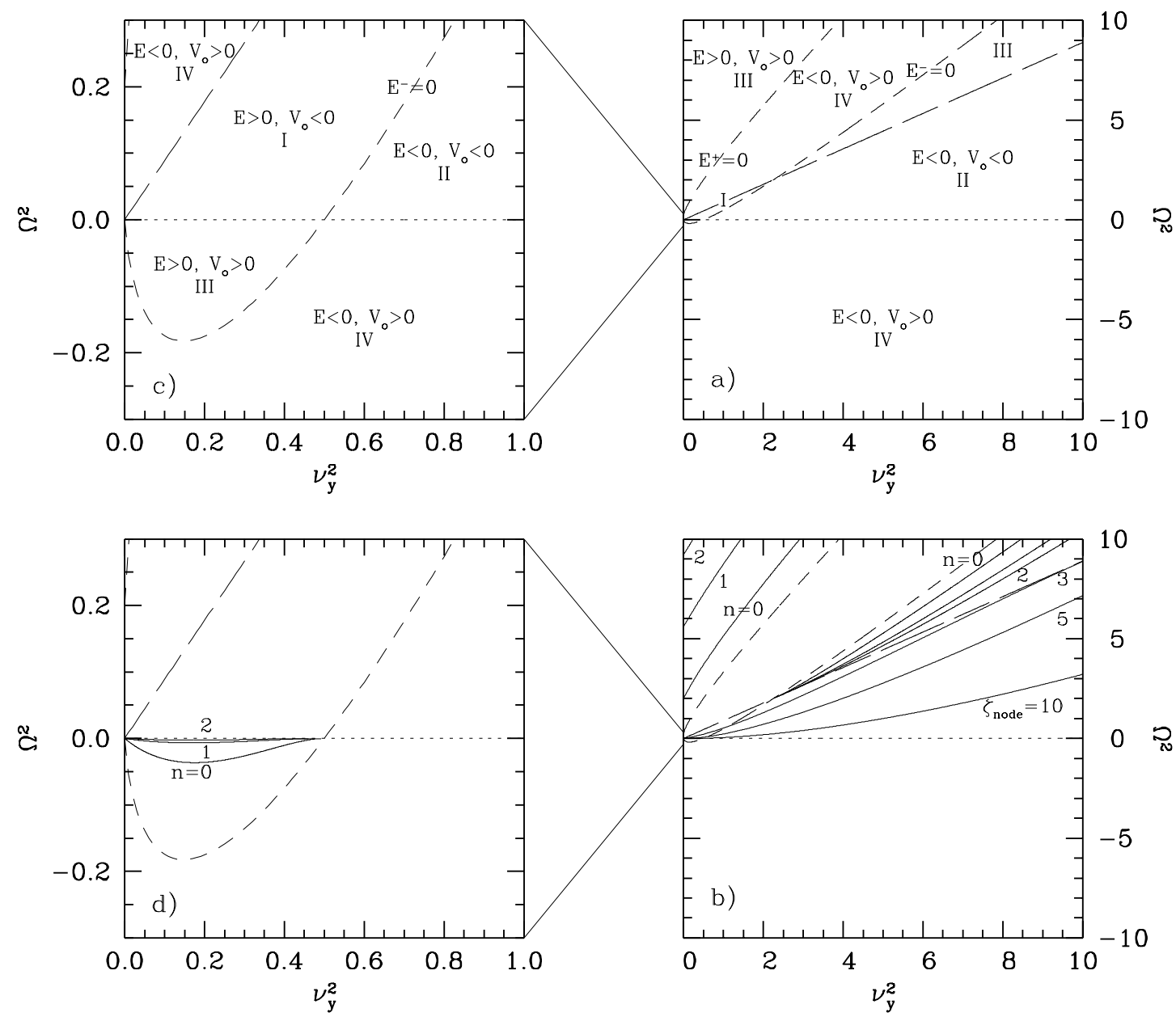

FIG. 7. - (a) Division of the phase space of $v_{y}^{2}$ and $\Omega^{2}$ into regions with the solutions of different types for a magnetized disk with $\alpha=1, \beta=0, \gamma=\gamma_{\mathrm{cr}}$, and $v_{x}=0$. The loci of $E=0$ (dashed lines), $V_{0}=0$ (dotted line), and $(2 \alpha+\gamma) \Omega^{2}-2 \alpha \gamma v_{y}^{2}=0$ (long-dashed line) are drawn. (b) Dispersion relations of the discrete family solutions with $n=0,1,2$ in the regions of type III, and the dispersion relations of the continuum family solutions with nodal points $\zeta_{\text {node }}=3,5,10$ in the regions of types I and II. (c) Magnification of the region of type III enclosed by loci of $E^{-}=0$ and $V_{0}=0$. (d) Dispersion relations of the discrete family solutions with $n=0,1$ and 2 in the magnified region of type III.

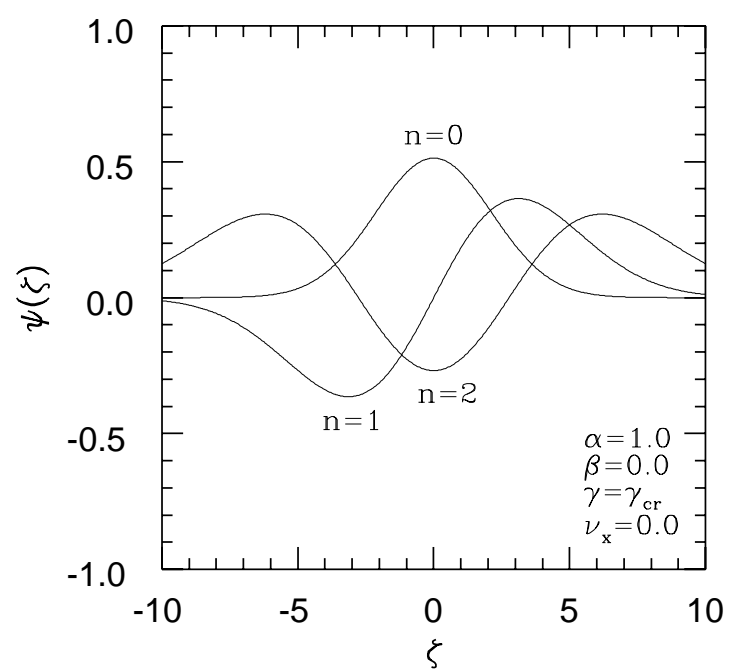

FIG. 8.-Eigenfunctions of the unstable discrete mode solutions with $n=0,1,2$ for $\alpha=1, \beta=0, \gamma=\gamma_{c r}$, and $v_{x}=0$. For a given $n$, the most unstable growth rate and its azimuthal wavenumber are used. other hand, if $\gamma_{\mathrm{cr}} \leq \gamma<\gamma_{\mathrm{int}}$, then the discrete mode is the only unstable one, so it determines the evolution of the medium.

If the scale height $(160 \mathrm{pc})$ and the velocity dispersion $(6.4$ $\mathrm{km} \mathrm{s}^{-1}$ ) of interstellar clouds are substituted for $H$ and $a_{s}$, respectively, then the growth time of the most unstable discrete solution with $n=0$ becomes $1.3 \times 10^{8} \mathrm{yr}$ at the wavelength of $2.4 \mathrm{kpc}$.

\subsubsection{Case of $v_{x} \neq 0$}

When $v_{x} \neq 0$, the Alfvén mode is no longer decoupled from the other modes. Figure $10 a$ presents the loci of $E=0$, $V_{0}=0,(2 \alpha+\gamma) \Omega^{2}-2 \alpha \gamma v_{y}^{2}=0$, and $\Omega^{2}-2 \alpha v_{y}^{2}=0$ for $\alpha=1, \beta=0, \gamma=1$, and $v_{x}=1$. In this general case, both loci of $E=0$ and $V_{0}=0$ are divided into positive and negative branches. The locus of $\Omega^{2}-2 \alpha v_{y}^{2}=0$ is drawn with a dot-dashed line. The six loci divide the phase space of $v_{y}^{2}$ and $\Omega^{2}$ into eight regions. Since $\gamma=1$, there is no region containing the unstable discrete mode solutions. The solutions of the continuum and discrete families identified in 

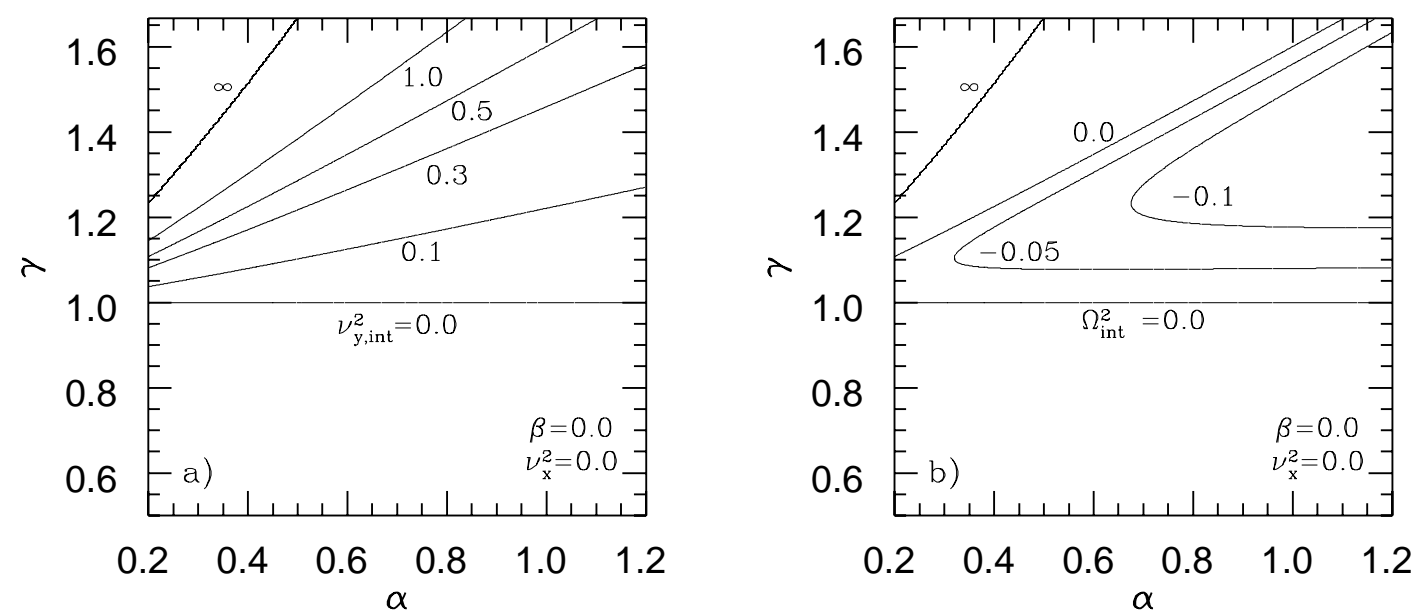

Fig. 9. - Contours of $(a)$ equi- $v_{y, \text { int }}^{2}$ and $(b)$ equi- $\Omega_{\text {int }}^{2}$ for $\beta=0$ on the $(\alpha, \gamma)$ plane. The point $\left(v_{y, \text { int }}^{2}, \Omega_{\text {int }}^{2}\right)$ is defined by the intersection of the two loci of $E^{-}=0$ and $V_{0}=0$. The intersection point exists only if $\gamma$ has a value in the region enclosed by the lines labeled with 0 and $\infty$.

Figures 6 and 7 are also found in the figure, and Figure $10 b$ shows their dispersion relations. In addition, we have a region of type II enclosed by the loci of $V_{0}^{+}=0$ and $\Omega^{2}$ $-2 \alpha v_{y}^{2}=0$. The solutions in this region are of the continuum family and always stable. Since one of the boundaries of this region corresponds to the dispersion relation of the Alfvén waves, we attribute its solutions to the Alfvén mode.

\section{SUMMARY AND DISCUSSION}

We have performed a linear stability analysis for magnetized disks under a linear gravity. The linearized perturbation equations have been reduced to an eigenvalue equation that has the same form as the Schrödinger equation with an energy $E$ and a potential $V_{0} \zeta^{2}$, where $E$ and $V_{0}$ are given by equations (2) and (3). By the signs of $E$ and $V_{0}$, the eigensolutions have been classified into two families: the continuum family for $V_{0}<0$ (independent of the sign of $E$ ) and the discrete family for $E>0$ and $V_{0}>0$.

Without a magnetic field, the solutions of the continuum

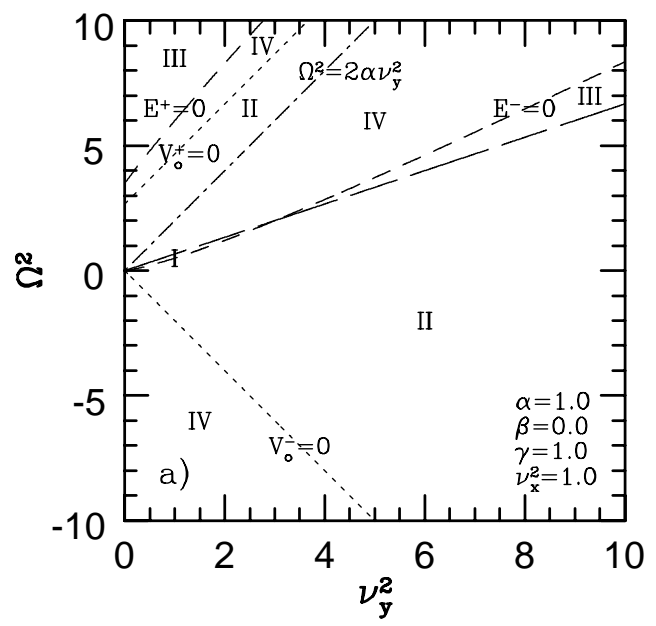

family are identified as the convective mode and those of the discrete family as the acoustic-gravity waves that were previously discussed by Nelson (1976). The convective mode develops into an instability if $\gamma<1$ (Ryu \& Goodman 1992).

With a magnetic field, the solutions of the continuum family are divided into two modes: a stable Alfvén mode and the original Parker mode. The Parker mode is a slow MHD mode modulated by the gravity and becomes unstable when the effective adiabatic index is smaller than the critical value $\gamma_{\mathrm{cr}}=(1+\alpha+\beta)^{2} /(1+3 \alpha / 2+\beta)$ for $v_{x}=$ 0 . The solutions of the discrete family are divided into three modes: a stable fast MHD mode modulated by the gravity, a stable slow MHD mode modulated by the gravity, and an unstable mode. The two stable discrete modes always exist, but the unstable discrete mode exists only if $1<\gamma<\gamma_{\text {int }}$, where $\gamma_{\text {int }}$ is a function of the parameters of the problem. The unstable discrete mode was first discovered by Giz \& Shu (1993).

Fig. 10.-(a) Division of the phase space of $v_{y}^{2}$ and $\Omega^{2}$ into regions with the solutions of different types for a magnetized disk with $\alpha=1, \beta=0, \gamma=1$, and $v_{x}^{2}=1$. The loci of $E=0$ (dashed lines), $V_{0}=0$ (dotted lines), $(2 \alpha+\gamma) \Omega^{2}-2 \alpha \gamma v_{y}^{2}=0$ (long-dashed line), and $\Omega^{2}-2 \alpha v_{y}^{2}=0$ (dot-dashed line) are drawn. (b) Dispersion relations of the discrete family solutions with $n=0,1,2$ in the regions of type III, and the dispersion relations of the continuum family solutions with nodal points $\zeta_{\text {node }}=3,5,10$ in the regions of types I and II. 
In the Galactic context, we discuss the possible roles of the unstable modes of the continuum and discrete solutions on the formation of giant molecular clouds and corrugations. For the scale height and the velocity dispersion of the cloud distribution, we take $160 \mathrm{pc}$ and $6.4 \mathrm{~km} \mathrm{~s}^{-1}$ (Falgarone \& Lequeux 1973). Then, the maximum growth time and the corresponding length of the continuum solution are $1.5 \times 10^{7} \mathrm{yr}$ and $340 \mathrm{pc}$, for the parameters of $\alpha=0.25, \beta=0.4, \gamma=1.0, v_{x}=0.0$ and $\zeta_{\text {node }}=3$. Here, the values of $\alpha$ and $\beta$ are the canonical ones of the interstellar medium (Spitzer 1978) and the chosen nodal point is about $500 \mathrm{pc}$ (Elmegreen 1982). On the basis of the time and length scales of the Parker instability under the uniform gravity, Mouschovias et al. (1974) preferred the interstellar medium behind Galactic shocks to the canonical interstellar medium, since the growth time of the Parker instability with the canonical parameters of the interstellar medium is larger than the lifetime of the giant molecular clouds, $3 \times 10^{7} \mathrm{yr}$ (Blitz \& Shu 1980). Under the linear gravity, the unstable modes of the continuum solution may, however, develop within the lifetime. Therefore, developments of the unstable modes of the continuum solution of the Parker instability under the linear gravity are not confined to spiral arms but are ubiquitous in the Galaxy.

We also suggest that the corrugations observed in our Galaxy and external galaxies may have been formed by the unstable discrete mode. For parameters, $\alpha=1, \beta=0, \gamma=$ $\gamma_{\text {cr }}$, and $v_{x}=0$, the growth time of the most unstable discrete solution with $n=0$ is $1.3 \times 10^{8} \mathrm{yr}$ at the wavelength of $2.4 \mathrm{kpc}$. This timescale is comfortably shorter than the age of the galaxies, and the length scale is consistent with the characteristic wavelength of the vertical structure of the Carina-Sagittarius spiral arm (Alfaro et al. 1992). Furthermore, the antisymmetric nature of the $n=0$ solution is also consistent with the corrugations found by Alfaro et al. (1992).

In this paper, we have neglected the effects of rotation on the Parker instability. Although the effects of differential rotation, which occurs in accretion disks as well as galactic disks, are difficult to work with analytically, those of rigidbody rotation can be included in the frame of the present work. The inclusion of rigid-body rotation is expected to have two major effects on our results. First, although it does not change the criterion of the instability, the inclusion of rigid-body rotation reduces the growth rate, as already pointed out in Shu (1974) and Zweibel \& Kulsrud (1975). Second, also as pointed out in Shu (1974), with rigid-body rotation the Lindblad oscillation enters into the problem, making the classification (for instance, the one in Fig. 10) more complicated. We are working on these, and the results will be reported in a separate paper.

At an early stage of this work J. K. was supported by a DAEWOO Post-Graduate Scholarship. The work of S. S. H. and J. K. was supported by the Seoul National University DAEWOO Research Fund. The work of D. R. was supported in part by the Seoam Scholarship Foundation. We are grateful to T. W. Jones and the referee, E. G. Zweibel, for comments on the manuscript.

\section{APPENDIX A}

\section{EQUILIBRIUM STATES AND LINEARIZED PERTURBATION EQUATIONS}

The MHD equations for gas with cosmic rays are given by

$$
\begin{gathered}
\frac{\partial \rho}{\partial t}+\nabla \cdot(\rho v)=0 \\
\rho\left(\frac{\partial v}{\partial t}+\boldsymbol{v} \cdot \nabla \boldsymbol{v}\right)=-\nabla p-\nabla p_{\mathrm{cr}}-\nabla \frac{B^{2}}{8 \pi}+\frac{1}{4 \pi} \boldsymbol{B} \cdot \nabla \boldsymbol{B}+\rho \boldsymbol{g} \\
\frac{\partial \boldsymbol{B}}{\partial t}=\nabla \times(\boldsymbol{v} \times \boldsymbol{B}) \\
\left(\frac{\partial}{\partial t}+\boldsymbol{v} \cdot \nabla\right)\left(\frac{p}{\rho^{\gamma}}\right)=0 \\
\boldsymbol{B} \cdot \nabla p_{\mathrm{cr}}=0
\end{gathered}
$$

where $p_{\text {cr }}$ is the cosmic-ray pressure.

For cosmic rays we use the equation that was employed in the study of the effect of rotation on the Parker instability by Shu (1974). It is valid on the assumption that cosmic rays adjust themselves to flows with an infinite speed along magnetic field lines. If the detail dynamics of cosmic rays is important, for instance, around the shocks where they are accelerated, then it can be treated either by the diffusion-advection equation such as derived by Skilling (1975) or by the two-fluid model (Drury \& Völk 1981). In the two-fluid model, cosmic rays are represented by a fluid with an adiabatic index between $4 / 3$ and $5 / 3$. With these more realistic treatments of cosmic rays, it was shown that fluids with cosmic rays are subject to cosmic-ray induced instabilities with a scale comparable to the diffusion length of cosmic rays (see, e.g., Drury \& Falle 1986; Zank, Axford, \& McKenzie 1990; Kang, Jones, \& Ryu 1992; Ryu, Kang, \& Jones 1993; Begelman \& Zweibel 1994). However, since here we are interested in the Parker instability, which occurs in a much larger scale, mainly by the coupling of magnetic field with gravity, the above simpler equation for cosmic rays should be enough.

To describe the local behavior of the Parker instability in magnetized disks, we introduce Cartesian coordinates $(x, y, z)$ whose directions are radial, azimuthal and vertical, respectively. We assume that the gravity, $g$, is vertical and is a linear 
function of $z, g=\left(0,0,-g^{\prime} z\right)$, and that the unperturbed magnetic field, $\boldsymbol{B}_{0}$, is a function of $z$ and has only an azimuthal component, $\boldsymbol{B}_{0}=\left[0, B_{0}(z), 0\right]$. Here, $g^{\prime}$ is a positive constant. We further assume that unperturbed gas is isothermal and that the ratio of its magnetic pressure to gas pressure, $\alpha$, and the ratio of its cosmic-ray pressure to gas pressure, $\beta$, are spatially constant, i.e., $p_{0}=a_{s}^{2} \rho_{0}, B_{0}^{2} /(8 \pi)=\alpha p_{0}$, and $p_{\text {cr,o }}=\beta p_{0}$, where $a_{s}$ is the isothermal sound speed and the quantities with subscript 0 indicate unperturbed quantities. Then, the vertical distribution of unperturbed quantities is

$$
\frac{\rho_{0}(z)}{\rho_{0}(0)}=\frac{p_{0}(z)}{p_{0}(0)}=\frac{p_{\mathrm{cr}, 0}(z)}{p_{\mathrm{cr}, 0}(0)}=\frac{B_{0}^{2}(z)}{B_{0}^{2}(0)}=\exp \left[-\frac{1}{2}\left(\frac{z}{H}\right)^{2}\right]
$$

where $H^{2} \equiv(1+\alpha+\beta) a_{s}^{2} / g^{\prime}$ and $\rho_{0}(0), p_{0}(0), p_{\mathrm{cr}, 0}(0), B_{0}(0)$ are the values of $\rho_{0}(z), p_{0}(z), p_{\mathrm{cr}, 0}(z), B_{0}(z)$ at $z=0$.

We perturb the above equilibrium state. The perturbed state is described by

$$
v ; \quad \rho=\rho_{0}+\delta \rho ; \quad p=p_{0}+\delta p ; \quad p_{\mathrm{cr}}=p_{\mathrm{cr}, 0}+\delta p_{\mathrm{cr}} ; \quad \boldsymbol{B}=B_{0} \hat{\boldsymbol{e}}_{y}+\delta \boldsymbol{B} .
$$

If we insert the quantities in equation (A7) into equations (A1)-(A5) and keep only the first-order terms of the perturbed variables, then we obtain the linearized perturbation equations

$$
\begin{gathered}
\frac{\partial \delta \rho}{\partial t}-\frac{\rho_{0}}{H^{2}} z v_{z}+\rho_{0}\left(\frac{\partial v_{x}}{\partial x}+\frac{\partial v_{y}}{\partial y}+\frac{\partial v_{z}}{\partial z}\right)=0 \\
\rho_{0} \frac{\partial v_{x}}{\partial t}+\frac{\partial \delta p}{\partial x}+\frac{\partial \delta p_{\mathrm{cr}}}{\partial x}+\frac{B_{0}}{4 \pi} \frac{\partial \delta B_{y}}{\partial x}-\frac{B_{0}}{4 \pi} \frac{\partial \delta B_{x}}{\partial y}=0 \\
\rho_{0} \frac{\partial v_{y}}{\partial t}+\frac{\partial \delta p}{\partial y}+\frac{\partial \delta p_{\mathrm{cr}}}{\partial y}+\frac{B_{0}}{8 \pi H^{2}} z \delta B_{z}=0 \\
\rho_{0} \frac{\partial v_{z}}{\partial t}+\frac{\partial \delta p}{\partial z}+\frac{\partial \delta p_{\mathrm{cr}}}{\partial z}-\frac{B_{0}}{8 \pi H^{2}} z \delta B_{y}-\frac{B_{0}}{4 \pi} \frac{\partial \delta B_{z}}{\partial y}+\frac{B_{0}}{4 \pi} \frac{\partial \delta B_{y}}{\partial z}+\frac{(1+\alpha+\beta) a_{s}^{2}}{H^{2}} z \delta \rho=0, \\
\frac{\partial \delta B_{x}}{\partial t}-B_{0} \frac{\partial v_{x}}{\partial y}=0, \\
\frac{\partial \delta B_{y}}{\partial t}+B_{0} \frac{\partial v_{x}}{\partial x}-\frac{B_{0}}{2 H^{2}} z v_{z}+B_{0} \frac{\partial v_{z}}{\partial z}=0 \\
\frac{\partial \delta B_{z}}{\partial t}-B_{0} \frac{\partial v_{z}}{\partial y}=0, \\
\frac{\partial \delta p}{\partial t}-\frac{p_{0}}{H^{2}} z v_{z}+\gamma p_{0}\left(\frac{\partial v_{x}}{\partial x}+\frac{\partial v_{y}}{\partial y}+\frac{\partial v_{z}}{\partial z}\right)=0 \\
\frac{\partial \delta p_{\mathrm{cr}}}{\partial t}-\frac{p_{\mathrm{cr}, 0}}{H^{2}} z v_{z}=0 .
\end{gathered}
$$

Since the coefficients of equations (A8)-(A16) do not depend explicitly on $x, y, t$, the perturbed quantities can be Fourier decomposed with respect to $x, y, t$. So the perturbed quantities have the following form:

$$
\delta Q(x, y, z ; t)=\delta Q(z) \exp \left(i \omega t-i k_{x} x-i k_{y} y\right),
$$

where $\omega$ is the angular frequency and $k_{x}$ and $k_{y}$ are the wavenumbers along the $x$ and $y$ directions. Inserting the above into equations (A8)-(A16), we get

$$
\begin{gathered}
i \omega \delta \rho-\frac{\rho_{0}}{H^{2}} z v_{z}+\rho_{0}\left(-i k_{x} v_{x}-i k_{y} v_{y}+\frac{d v_{z}}{d z}\right)=0, \\
i \omega \rho_{0} v_{x}-i k_{x} \delta p-i k_{x} \delta p_{\mathrm{cr}}-i k_{x} \frac{B_{0}}{4 \pi} \delta B_{y}+i k_{y} \frac{B_{0}}{4 \pi} \delta B_{x}=0, \\
i \omega \rho_{0} v_{y}-i k_{y} \delta p-i k_{y} \delta p_{\mathrm{cr}}+\frac{B_{0}}{8 \pi H^{2}} z \delta B_{z}=0, \\
i \omega \rho_{0} v_{z}+\frac{d \delta p}{d z}+\frac{d \delta p_{\mathrm{cr}}}{d z}-\frac{B_{0}}{8 \pi H^{2}} z \delta B_{y}+i k_{y} \frac{B_{0}}{4 \pi} \delta B_{z}+\frac{B_{0}}{4 \pi} \frac{d \delta B_{y}}{d z}+\frac{(1+\alpha+\beta) a_{s}^{2}}{H^{2}} z \delta=0, \\
i \omega \delta B_{x}+i k_{y} B_{0} v_{x}=0, \\
i \omega \delta B_{y}-i k_{x} B_{0} v_{x}-\frac{B_{0}}{2 H^{2}} z v_{z}+B_{0} \frac{d v_{z}}{d z}=0,
\end{gathered}
$$




$$
\begin{gathered}
i \omega \delta B_{z}+i k_{y} B_{0} v_{z}=0, \\
i \omega \delta p-\frac{p_{0}}{H^{2}} z v_{z}+\gamma p_{0}\left(-i k_{x} v_{x}-i k_{y} v_{y}+\frac{d v_{z}}{d z}\right)=0, \\
i \omega \delta p_{\mathrm{cr}}-\frac{p_{\mathrm{cr}, 0}}{H^{2}} z v_{z}=0 .
\end{gathered}
$$

The above nine equations (eqs. [A18]-[A26]) with nine unknowns $\left(\delta \rho, v_{x}, v_{y}, v_{z}, \delta B_{x}, \delta B_{y}, \delta B_{z}, \delta p\right.$, and $\left.\delta p_{\mathrm{cr}}\right)$ can be combined into a single second-order differential equation for one unknown:

$$
\begin{aligned}
\frac{d^{2} v_{z}}{d z^{2}} & -\frac{z}{H^{2}} \frac{d v_{z}}{d z}+\frac{\omega^{4} / a_{s}^{4}-\left[(1+\alpha+\beta) / H^{2}+(2 \alpha+\gamma)\left(k_{x}^{2}+k_{y}^{2}\right)\right] \omega^{2} / a_{s}^{2}+2 \alpha \gamma\left(k_{x}^{2}+k_{y}^{2}\right) k_{y}^{2}}{(2 \alpha+\gamma) \omega^{2} / a_{s}^{2}-2 \alpha \gamma k_{y}^{2}} v_{z} \\
& +\left\{\frac{2 \alpha(1+\alpha+\beta) k_{x}^{2} \omega^{2} / a_{s}^{2}}{\left(\omega^{2} / a_{s}^{2}-2 \alpha k_{y}^{2}\right)\left[(2 \alpha+\gamma) \omega^{2} / a_{s}^{2}-2 \alpha \gamma k_{y}^{2}\right]}-\frac{(1+\alpha+\beta)(1+\alpha+\beta-\gamma)\left(k_{x}^{2}+k_{y}^{2}\right)}{(2 \alpha+\gamma) \omega^{2} / a_{s}^{2}-2 \alpha \gamma k_{y}^{2}}\right\} \frac{z^{2}}{H^{4}} v_{z}=0 .
\end{aligned}
$$

With $\Psi$ defined as

$$
v_{z}=\Psi \exp \left[\frac{1}{4}\left(\frac{z}{H}\right)^{2}\right]
$$

the equation reduces to a second-order differential equation without the first-order derivative term

$$
\begin{aligned}
\frac{d^{2} \Psi}{d z^{2}} & +\left\{\frac{1}{2 H^{2}}+\frac{\omega^{4} / a_{s}^{4}-\left[(1+\alpha+\beta) / H^{2}+(2 \alpha+\gamma)\left(k_{x}^{2}+k_{y}^{2}\right)\right] \omega^{2} / a_{s}^{2}+2 \alpha \gamma\left(k_{x}^{2}+k_{y}^{2}\right) k_{y}^{2}}{(2 \alpha+\gamma) \omega^{2} / a_{s}^{2}-2 \alpha \gamma k_{y}^{2}}\right\} \Psi \\
& -\left\{\frac{1}{4}-\frac{2 \alpha(1+\alpha+\beta) k_{x}^{2} \omega^{2} / a_{s}^{2}}{\left(\omega^{2} / a_{s}^{2}-2 \alpha k_{y}^{2}\right)\left[(2 \alpha+\gamma) \omega^{2} / a_{s}^{2}-2 \alpha \gamma k_{y}^{2}\right]}+\frac{(1+\alpha+\beta)(1+\alpha+\beta-\gamma)\left(k_{x}^{2}+k_{y}^{2}\right)}{(2 \alpha+\gamma) \omega^{2} / a_{s}^{2}-2 \alpha \gamma k_{y}^{2}}\right\} \frac{z^{2}}{H^{4}} \Psi=0 .
\end{aligned}
$$

We define the dimensionless variables by

$$
\Omega \equiv \omega H / a_{s}, v_{x} \equiv k_{x} H, v_{y} \equiv k_{y} H, \psi \equiv \Psi / a_{s}, \zeta \equiv z / H .
$$

Then, the combined perturbation equation becomes

$$
\frac{d^{2} \psi}{d \zeta^{2}}+\left(E-V_{0} \zeta^{2}\right) \psi=0
$$

where $E$ and $V_{0}$ are given by

$$
\begin{gathered}
E \equiv \frac{1}{2}+\frac{\Omega^{4}-\left[(1+\alpha+\beta)+(2 \alpha+\gamma)\left(v_{x}^{2}+v_{y}^{2}\right)\right] \Omega^{2}+2 \alpha \gamma\left(v_{x}^{2}+v_{y}^{2}\right) v_{y}^{2}}{(2 \alpha+\gamma) \Omega^{2}-2 \alpha \gamma v_{y}^{2}}, \\
V_{0} \equiv \frac{1}{4}-\frac{2 \alpha(1+\alpha+\beta) v_{x}^{2} \Omega^{2}}{\left(\Omega^{2}-2 \alpha v_{y}^{2}\right)\left[(2 \alpha+\gamma) \Omega^{2}-2 \alpha \gamma v_{y}^{2}\right]}+\frac{(1+\alpha+\beta)(1+\alpha+\beta-\gamma)\left(v_{x}^{2}+v_{y}^{2}\right)}{(2 \alpha+\gamma) \Omega^{2}-2 \alpha \gamma v_{y}^{2}}
\end{gathered}
$$

(eqs. [A32] and [A33] are the same as eqs. [2] and [3], respectively).

\section{APPENDIX B}

\section{METHOD TO COMPUTE THE DISPERSION RELATIONS OF THE CONTINUUM FAMILY SOLUTIONS}

For given $\alpha, \beta$, and $\gamma, E$ and $V_{0}$ in equations (A32) and (A33) are functions of $v_{x}^{2}, v_{y}^{2}$, and $\Omega^{2}$. Thus, getting the dispersion relations is equivalent to finding the values of $v_{x}^{2}, v_{y}^{2}$, and $\Omega^{2}$, which satisfy the boundary conditions imposed on equation (A31). We consider the upper boundary condition $\psi=0$ at $\zeta=\zeta_{\text {node }}$ and the lower boundary condition $\psi(0)=0$ or $d \psi(0) / d \zeta=0$ at the midplane. Here, $\zeta_{\text {node }}$ is the nearest nodal point from the midplane. The condition $\psi(0)=0$ does not allow gas to cross the midplane. We call the solutions that satisfy this condition the symmetric mode. On the other hand, the condition $d \psi(0) / d \zeta=0$ allows gas to cross the midplane. We call the solutions that satisfy this second condition the antisymmetric mode.

We introduce an index, $i$, and define

$$
\zeta_{i} \equiv(i-1) \Delta \zeta, \quad \Delta \zeta \equiv \frac{\zeta_{\text {node }}}{N-1},
$$

where $N$ is the total number of points in the interval $\left[0, \zeta_{\text {node }}\right]$. With a central difference scheme, the finite-difference representation of the perturbation equation (A31) is

$$
\psi_{i-1}+\left[\Delta \zeta^{2}\left(E-V_{0} \zeta_{i}^{2}\right)-2\right] \psi_{i}+\psi_{i+1}=0
$$


where $i$ runs from 3 to $N-2$ for the symmetric mode and from 2 to $N-2$ for the antisymmetric mode. Because $\psi_{N}=0$ at the upper boundary, the finite-difference equation at the point $i=N-1$ is

$$
\psi_{N-2}+\left[\Delta \zeta^{2}\left(E-V_{0} \zeta_{N-1}^{2}\right)-2\right] \psi_{N-1}=0
$$

For the symmetric mode with $\psi_{1}=0$, we use the finite-difference equation at the point of $i=2$,

$$
\left[\Delta \zeta^{2}\left(E-V_{0} \zeta_{2}^{2}\right)-2\right] \psi_{2}+\psi_{3}=0 .
$$

For the antisymmetric mode with $\psi_{0}=\psi_{2}$, we use the finite-difference equation at the point of $i=1$,

$$
\left[\Delta \zeta^{2}\left(E-V_{0} \zeta_{1}^{2}\right)-2\right] \psi_{1}+2 \psi_{2}=0 .
$$

We can write the simultaneous equations for $\psi_{i}$ in a matrix form,

$$
M \psi=\left(\begin{array}{cccccc}
\Delta \zeta^{2}\left(E-V_{0} \zeta_{s}^{2}\right)-2 & * & \ldots & 0 & 0 & 0 \\
1 & \Delta \zeta^{2}\left(E-V_{0} \zeta_{s-1}^{2}\right)-2 & 1 & \ldots & 0 & 0 \\
\vdots & \vdots & \vdots & & \vdots & \vdots \\
0 & 0 & \ldots & 1 & \Delta \zeta^{2}\left(E-V_{0} \zeta_{N-2}^{2}\right)-2 & 1 \\
0 & 0 & 0 & \ldots & 1 & \Delta \zeta^{2}\left(E-V_{0} \zeta_{N-1}^{2}\right)-2
\end{array}\right)\left(\begin{array}{c}
\psi_{s} \\
\psi_{s+1} \\
\vdots \\
\psi_{N-2} \\
\psi_{N-1}
\end{array}\right)=\left(\begin{array}{c}
0 \\
0 \\
\vdots \\
0 \\
0
\end{array}\right)
$$

where $s$ is 2 for the symmetric mode and 1 for the antisymmetric mode, and the asterisk is 1 for the symmetric mode and 2 for the antisymmetric mode. Nontrivial solutions exist only if the determinant of the tri-diagonal matrix $M$ is equal to zero. For given $\alpha, \beta$, and $\gamma$, the coefficients of the matrix $M$ are functions of $v_{x}^{2}, v_{y}^{2}$, and $\Omega^{2}$. Thus, the problem becomes an eigenvalue problem, i.e., with given $v_{x}^{2}$ and $v_{y}^{2}$, we should find $\Omega^{2}$, which makes the determinant of $M$ equal to zero. We used the Gauss-Seidel method for it.

\section{REFERENCES}

Abramowitz, M., \& Stegun, I. A. 1970, Handbook of Mathematical Functions (New York: Dover)

Alfaro, E. J., Cabrera-Caño, J., \& Delgado, A. J. 1992, ApJ, 399, 576

Appenzeller, I. 1974, A\&A, 36, 99

Begelman, M. C., \& Zweibel, E. G. 1994, ApJ, 431, 689

Blitz, L. , \& Shu, F. H. 1980, ApJ, 238, 148

Drury, L. O. , \& Falle, S. A. E. G. 1986, MNRAS, 223, 353

Drury, L. O., \& Völk, H. J. 1981, ApJ, 248, 344

Elmegreen, B. G. 1982, ApJ, 253, 634

Falgarone, E., \& Lequeux, J. 1973, A\&A, 25, 253

Foglizzo, T., \& Tagger, M. 1994, A\&A, 287, 297

Florido, E., Battaner, E., Prieto, M., Mediavilla, E. , \& Sanchez-Saavedra, M. L. 1991, MNRAS, 251, 193

Giz, A. T. , \& Shu, F. H. 1993, ApJ, 404, 185

Gomez de Castro, A. I., \& Pudritz, R. E. 1992, ApJ, 395, 501

Hanawa, T., Matsumoto, R., \& Shibata, K. 1992, ApJ, 393, L71

Horiuchi, T., Matsumoto, R., Hanawa, T., \& Shibata, K. 1988, PASJ, 40, 147

Kaisig, M., Tajima, T., Shibata, K., Nozawa, S., \& Matsumoto, R. 1990, ApJ, 358, 698

Kang, H., Jones, T. W. , \& Ryu, D. 1992, ApJ, 385, 193

Lockman, F. J. 1977, AJ, 82, 408

Malhotra, S. 1994, ApJ, 433, 687

Matsumoto, R., Horiuchi, T., Shibata, K., \& Hanawa, T. 1988, PASJ, 40, 171
Mouschovias, T. C. 1974, ApJ, 192, 37

Mouschovias, T. C. , Shu, F. H. , \& Woodward, P. R. 1974, A\&A, 33, 73

Nelson, A. H. 1976, MNRAS, 174, 661

Nozawa, S., Shibata, K., Matsumoto, R., Sterling, A. C., Tajima, T., Uchida, Y., Ferrari, A. , \& Rosner, R. 1992, ApJS, 78, 267

Oort, J. H. 1965, in Galactic Structure, ed. A. Blaauw \& M. Schmidt (Chicago: Univ. Chicago Press), 455

Parker, E. N. 1966, ApJ, 145, 811

. 1967, ApJ, 149, 535

Quiroga, R. J., \& Schlosser, W. 1977, A\&A, 57, 455

Ryu, D., \& Goodman, J. 1992, ApJ, 388, 438

Ryu, D., Kang, H. , \& Jones, T. W. 1993, ApJ, 405, 199

Shibata, K. , \& Matsumoto, R. 1991, Nature, 353, 633

Shibata, K., Tajima, T., Matsumoto, R., Horiuchi, T., Hanawa, T., Rosner, R., \& Uchida, Y. 1989a, ApJ, 338, 471

Shibata, K., Tajima, T., Steinolfson, R. S., \& Matsumoto, R. 1989b, ApJ, 345,584

Shu, F. H. 1974, A\&A, 33, 55

Skilling, J. 1975, MNRAS, 172, 557

Spicker, J., \& Feitzinger, J. V. 1986, A\&A, 163, 43

Spitzer, L., Jr. 1978, Physical Processes in the Interstellar Medium (New York: Wiley), 226

Tout, C. A. , \& Pringle, J. E. 1992, MNRAS, 259, 604

Zank, G. P., Axford, W. I. , \& McKenzie, J. F. 1990, A\&A, 233, 275

Zweibel, E. G. , \& Kulsrud, R. M. 1975, ApJ, 201, 63 